\title{
Systemic Quinolones and Risk of Retinal Detachment II: Systematic Review of Clinical Trials
}

\section{Mohamed Kadry Taher 1,2,3, Mohamed Habsah', ${ }^{2,4}$ Lise M Bjerre, ${ }^{2,5,6}$, Franco Momoli, ${ }^{2,3,7}$, Donald Mattison $^{1,2,3}$ and Daniel Krewski, ${ }^{1,2,3}$}

${ }^{1}$ McLaughlin Centre for Population Health Risk Assessment, Faculty of Medicine, University of Ottawa, Canada

${ }^{2}$ School of Epidemiology and Public Health, University of Ottawa, Ottawa, ON, Canada

${ }^{3}$ Risk Sciences International, Canada

${ }^{4}$ Canadian Red Cross, Ottawa, ON, Canada

${ }^{5}$ Department of Family Medicine, University of Ottawa, Canada

${ }^{6}$ Montfort Knowledge Institute, Ottawa, ON, Canada

${ }^{7}$ Children's Hospital of Eastern Ontario Research Institute, Canada

*Corresponding author: Dr. Mohamed Kadry Taher, McLaughlin Centre for Population Health Risk Assessment, Faculty of Medicine, University of Ottawa, 600 Peter Morand Crescent, Room 216, Ottawa, ON, K1G 5Z3, Canada, Tel: +1-(613)562-5381, Fax: +1-(613)-562-5380

\begin{abstract}
Introduction: Quinolones represent a popular group of antibiotics that is favored by physicians due to their potency, broad-spectrum, unique mechanism of action, and reasonable safety. Concerns have been raised about a possible association between quinolones and retinal detachment (RD). Despite the emergence of resistance and availability of other treatment alternatives, quinolones remain a popular choice among physicians worldwide.
\end{abstract}

Areas covered: This systematic review examined all original clinical trials in which a quinolone antibiotic was tested or used as a comparator, for evidence on occurrences of retinal detachment. We examined four major bibliographic databases, eight clinical trial registries, and grey literature sources including international conference proceedings, drug review networks and databases of pharmaceutical companies for ongoing or unpublished studies. This systematic review has been conducted according to the PRISMA guidelines and has been registered in PROSPERO: CRD42020148747.

Expert opinion: We identified 1,554 original studies that examined quinolone antibiotics between 1974-2020 on participants of different ages, ethnic/racial backgrounds, and health status. Among the 145 eligible trials identified, no cases of retinal detachment were reported. There was no evidence from clinical trials to question systemically administered quinolone antibiotics as a cause of retinal detachment in persons with no history of eye diseases.

\section{Keywords}

Clinical trials, Drug safety, Pharmacovigilance, Quinolones, Retinal detachment, Systematic review

\section{Introduction}

Quinolones are synthetic antibiotics introduced in the mid-1960s that continue to gain popularity on a global scale [1]. The popularity of quinolones may be attributed to their potency, broad spectrum, unique mechanism of action and strong pharmacologic profile relative to other classes of antibiotics [17]. Despite the occurrence of adverse reactions, emergence of resistance, and the availability of other treatment alternatives [1], quinolones continue to be widely prescribed. Whereas quinolones are generally considered as having a reasonable safety profile, with most adverse reactions being mild to moderate and self-limiting, some quinolones have been withdrawn from the market and others have sustained restricted 
usage advisories [7-10].

A number of studies reported ocular adverse reactions in association with use of quinolone antibiotics, including retinal hemorrhage [11], macular degeneration [12], corneal perforation [13], and optic neuropathy [14]. These adverse reactions were reported in association with either systemic or topical quinolone formulations.

The present review focuses on the occurrence of retinal detachment within thirty days following the administration of a single systemically administered quinolone antibiotic. Retinal detachment (RD) is a serious condition involving the separation of the retinal layer of the eye from its underlying tissues with the subsequent loss of blood and oxygen supply, leading to loss of vision in the affected eye(s) if left untreated [15].

Quinolones are known for having a destructive effect on connective tissues [16], which led to a class warning for association with adverse reactions such as tendon rupture $[11,17]$. This may represent a plausible mechanism for causing retinal detachment in humans [18] and animal [19]. Some FDA reports and other epidemiologic studies reported an increased risk of retinal detachment with use of different formulations of quinolones [18,20-35].

We conducted this systematic review of clinical trials as part of a multipronged strategy to conclusively verify such an association. The first part provides summary of evidence based on data mining of reports recorded in the US FDA Adverse Event Reporting System (FAERS) [36]. The third part involves a case-control study based on a major US electronic health records database [37].

\section{Methods}

\section{Review strategy}

We implemented a comprehensive, multi-step search strategy to identify original studies that examined the association between quinolone antibiotics and the risk of retinal detachment. The search was conducted in accordance with the PRISMA guidelines (Preferred Reporting Items for Systematic Reviews and MetaAnalyses), and following the specific guidance provided by the Cochrane Collaboration [38]. The search strategy was designed and implemented on March 8-9, 2019, and updated on August 2-4, 2020 to identify all relevant original studies related to each of the 30 members of the quinolone class of antibiotics shown in Table 1.
We searched major bibliographic databases and clinical trial registries as well as major grey literature sources such as drug review networks and databases of pharmaceutical companies, relevant national and international agencies and international conference proceedings. Experts were consulted on all components of the review strategy and implementation. We also inspected the bibliographies of examined studies for additional relevant studies not already identified via the original search. This review has been registered in the international prospective register of systematic reviews PROSPERO under reference number CRD42020148747.

\section{Criteria for considering studies for this review}

With our focus on the safety of quinolone antibiotics within the present review, the outcome measure of interest is the number of de novo cases of retinal detachment within 30 days of systemic administration of a quinolone antibiotic in patients with no prior history of eye disease. Clinical trial eligible for inclusion in this review comprise those examining a single systemicallyadministered quinolone antibiotic compared to a placebo, another non-quinolone antibiotic or nonquinolone-containing antibiotic combination. Trials with multiple arms, which include a single quinolone in one arm and a placebo in one of the other arms, would be considered eligible for inclusion.

Trials where participants had prior or existing eye diseases; trials testing topical/non-systemically administered quinolones, a combination of quinolones, or a combination of quinolones and other drugs; and trials testing efficacy of different doses of a quinolone antibiotic, or testing a quinolone against another quinolone, were excluded from this review. Crossover trials were also excluded to avoid possible carryover effects from the initial intervention. Non-original studies, observational studies and trials where no results were available were also excluded. We enforced no restrictions on participants' age, sex, racial/ethnicity or any other background characteristics.

\section{Search methods for identification of studies}

Bibliographic databases searched included Medline (Ovid MEDLINE ${ }^{\circledast}$ In-Process \& Other Non-Indexed Citations and Ovid MEDLINE ${ }^{\circledR} 1946$ to Present), EMBASE (Embase Classic + Embase 1947 to 2017 May 02), Cochrane Central Register of Controlled Trials (CENTRAL), and CINAHL (Cumulative Index to Nursing

Table 1: Four generations of quinolone antibiotics.

\begin{tabular}{|l|l|}
\hline Generation & Quinolone Antibiotic \\
\hline First & Nalidixic acid, cinoxacin, flumequine, oxolinic acid, piromidic acid, pipemidic acid, rosoxacin \\
\hline Second & Lomefloxacin, norfloxacin, ciprofloxacin, ofloxacin, fleroxacin, pefloxacin, rufloxacin \\
\hline Third & Levofloxacin, sparfloxacin, temafloxacin, grepafloxacin, balofloxacin, pazufloxacin, tosufloxacin \\
\hline Fourth & $\begin{array}{l}\text { Moxifloxacin, gemifloxacin, trovafloxacin, gatifloxacin, clinafloxacin, garenoxacin, sitafloxacin, prulifloxacin, } \\
\text { finafloxacin }\end{array}$ \\
\hline
\end{tabular}


and Allied Health Literature).

Search terms included medical subject headings and text word search for quinolone antibiotics both as a class and as individual agents, in addition to retinal detachment as the outcome of interest. The search integrated a specialized search algorithm with reported high efficiency for identifying clinical trials in MEDLINE Ovid, EMBASE and CINAHL [39]. We also used another specialized algorithm for identifying adverse reactions [40] with the MEDLINE and EMBASE databases only. No language, time or other filters were used to limit the search output. A detailed description of the search terms used is provided in Supplementary Material I. A complete listing of studies considered, with reasons for exclusion of studies not meeting our inclusion criteria, is provided in Supplementary Material II.

An extensive search was conducted to identify all clinical trials conducted on any of the quinolone antibiotics documented in any of the major international clinical trial registries, including the World Health Organization Clinical Trials Registry (ICTRN), United States Clinical Trials Database (Clinicaltrials.gov), as well as similar registries from the European Union, United Kingdom, Netherlands, Japan, China, South Korea, India, Iran, Australia and South Africa. We also searched major grey literature sources including international conference proceedings, drug review networks and databases of pharmaceutical companies for ongoing or unpublished studies.

Duplicate screening of titles and abstracts (Level 1) and full-text examination (Level 2) were performed independently by two reviewers ( $\mathrm{MT}$ and $\mathrm{MH}$ ) to identify studies eligible for inclusion in the review, based on the predetermined eligibility criteria. Conflicts identified in each step were resolved via consensus between the reviewers or via a panel of senior scientists (FM, LB, DM, DK), prior to moving to the next step.

In the event where multiple publications report on an original study/trial, only the primary record was assessed in the review. All studies which reported on, or have been indexed to a specific original study/trial, were listed under the original study/trial record. Maximum diligence was adopted to include all publications reporting on each of the included clinical trials. A complete listing of original studies and associated publications is included in Supplementary Material III.

\section{Data collection and analysis}

We collated the references identified from all sources using EndNote [41] reference management application version 8.2, which was used to flag potential duplicates, with manual resolution employed to remove actual duplicates.

We used Review Manager application version 5.3 [42] to collate and classify all examined studies, tabulate the reasons for study exclusion, and compile the information required for generating the PRISMA flow diagram [43].

Data abstraction spreadsheets were developed to abstract the following information: study (design, year, country), tested quinolone antibiotic (name, dose, route of administration, frequency, duration), control agent/group (type, name, dose, route of administration, frequency, duration), participant characteristics (total sample size, age, sex, race), comorbidities (prior eye diseases/conditions, diabetes, alcohol abuse), treatment period and follow-up (average, range, years), main result(s), and authors' reported conclusion. Reviewers' comments, if any, are also included.

\section{Results}

\section{Flow of studies}

The search strategy resulted in retrieval of 3,461 records, including 3,430 records from bibliographic databases and clinical trial registries, 30 records from the databases of pharmaceutical companies, and 1 record from an international conference. Electronic and manual de-duplication resulted in removal of 787 records. Examined studies spanned across 5 decades (1974-2020), peaking between 2008-2016. These studies were conducted in many countries, with participants from all sexes, ethnicities, age groups and with different types and levels of comorbidity.

Title and abstract screening of 2,674 records resulted in the exclusion of 147 studies due to irrelevancy of exposure $(n=23)$ or publication type $(n=124)$, leaving 2,527 studies for full text examination. An additional 181 trials were not published at the time of completion of this review were also excluded. Upon full-text examination, we were able to identify 1,554 original and 973 nonoriginal records (duplicate reports on original studies). Only 145 original trials were found to meet the eligibility criteria; however, none of them reported a single case of retinal detachment in persons with otherwise healthy eyes. An additional 1,409 original studies were excluded for different reasons (see Supplementary Material II for reasons for excluding studies). A detailed PRISMA flow diagram for studies examined in this review [43] is shown in Figure 1.

\section{Characteristics of examined studies}

None of the identified original clinical trials that met the eligibility criteria in our review reported any cases of retinal detachment during the conduct of these clinical trials. Therefore, we were unable to retain any studies for further qualitative or quantitative analyses. Whenever a study had more than one reason for exclusion, we reported the study using the most compelling reason. A summary list of the reasons for exclusion of all studies examined in this review is shown in Table 2. A detailed listing of the reason of exclusion 


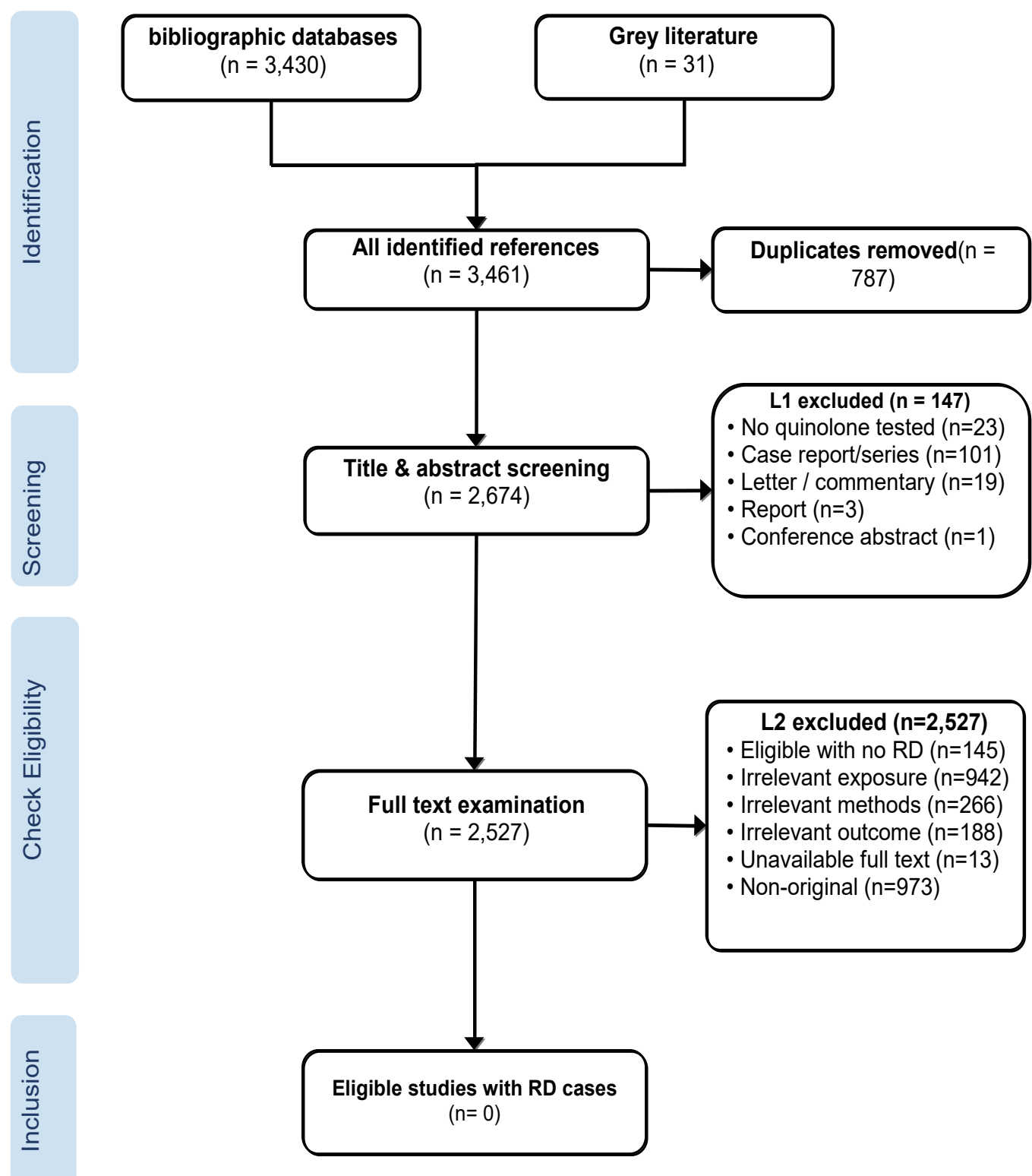

Figure 1: PRISMA flow diagram for studies conducted on/using quinolone antibiotics.

for each study and the full citations of original and supporting studies are provided in Supplementary Material II and Supplementary Material III, respectively.

\section{Discussion}

This review covers all publicly available original, peer-reviewed publications, as well as data from clinical trial registries and other grey literature sources for unpublished evidence that were conducted using quinolone antibiotics. There were no reports of retinal detachment in any study participants, with no history of eye diseases, within thirty days of receiving treatment with a single systemically administered quinolone antibiotic in any of the 145 studies included in this review.

Considering its serious nature, the occurrence of retinal detachment would mandate stoppage of treatment and transfer of participant to a hospital/ emergency room setting for an immediate medical/ surgical intervention to avoid permanent visual loss. Therefore, it is extremely unlikely that there were any undetected/unreported cases of such an adverse reaction.

Clinical trials represent the most rigorous assessment of human drug safety, which is used to examine the association between a specific $\operatorname{drug}(\mathrm{s})$ and specific adverse reactions (ADRs) $[44,45]$. However, clinical trials may be subject to a lack of representativeness since the characteristics of the selected participants will not necessarily mirror the general population with regards to demographics and other relevant risk factors that may impact how the drug works under reallife situations [44-48]. Because clinical trials typically involve a limited number of patients, it is possible that they might miss rare ADRs such as retinal detachment $[44,45,47,49,50]$.

To the best of our knowledge, this is the first 
Table 2: Reasons for study exclusion.

\begin{tabular}{|c|c|c|}
\hline Exclusion group & Reason for exclusion & Records \\
\hline \multicolumn{3}{|c|}{ Level 1: Title and abstract screening $(n=147)$} \\
\hline Irrelevant exposure & Irrelevant (no quinolone tested) & 23 \\
\hline \multirow[t]{4}{*}{ Irrelevant publication type } & Case report/case series & 101 \\
\hline & Letter/commentary & 19 \\
\hline & Report & 3 \\
\hline & Conference abstract & 1 \\
\hline \multicolumn{3}{|c|}{ Level 2: Full-text examination $(n=2,527)$} \\
\hline \multirow[t]{6}{*}{ Irrelevant exposure } & Quinolone combined with other drugs & 294 \\
\hline & Cross-over trial & 196 \\
\hline & Trial with multiple arms & 150 \\
\hline & Quinolone in a topical formulation & 129 \\
\hline & Trial with a single arm & 100 \\
\hline & Quinolones on multiple arms & 73 \\
\hline \multirow[t]{3}{*}{ Irrelevant methods } & Observational study & 147 \\
\hline & Review article & 102 \\
\hline & Not a clinical trial & 17 \\
\hline \multirow[t]{2}{*}{ Irrelevant outcome } & No results available & 181 \\
\hline & Current or prior eye disease & 7 \\
\hline Unavailable full-text & Reference could not be retrieved & 13 \\
\hline Non-original & Additional reports on original studies & 973 \\
\hline Eligible trials with no RD & Eligible trial with no reporting on $\mathrm{RD}$ & 145 \\
\hline
\end{tabular}

systematic review that examined all original studies that investigate the association of systemically administered quinolone antibiotics with the risk of retinal detachment.

\section{Conclusion}

Although the lack of reporting of a single case of retinal detachment in all trials examined in this review is encouraging, the possibility that use of quinolones could increase the risk of retinal detachment in the general population under real world conditions of use cannot be ruled out. Observational Pharmacovigilance studies based on large and diverse patient populations are needed to confidently confirm or reject an association between use of quinolones and increased risk of retinal detachment.

\section{Funding}

This research was supported in part with funding from the McLaughlin Centre for Population Health Risk Assessment at the University of Ottawa. D. Krewski is the Natural Sciences and Engineering Research Council of Canada Chair in Risk Science at the University of Ottawa.

\section{Author Contributions}

The primary author, Mohamed Taher, designed and implemented the review strategy including search methodology, design of study screening and data abstraction forms, reference collation and full-text acquisition, screening and examination of the identified references, data abstraction and drafting of this manuscript. Mohamed Habsah independently assessed all studies examined in this review. Lise Bjerre, Franco Momoli, Donald Mattison and Daniel Krewski provided guidance and feedback on all aspects of the review design and implementation, as well as critical review the manuscript.

\section{Declaration of Interest}

All authors who contributed to both this study and manuscript report no conflict of interest in relation to the planning for and conducting this study as well as production of this manuscript.

\section{References}

1. Liu $H(2010)$ Safety profile of the fluoroquinolones: Focus on levofloxacin. Drug Safety 33: 353-369.

2. (2014) Antibacterial Drugs Market Expected to Reach USD 45.09 Billion Globally in 2019: Transparency Market Research. Cision PR Newswire.

3. Appelbaum PC, Hunter PA (2000) The fluoroquinolone antibacterials: Past, present and future perspectives. Int $\mathrm{J}$ Antimicrob Agents 16: 5-15.

4. Emmerson AM, Jones AM (2003) The quinolones: Decades of development and use. J Antimicrob Chemother 51: 1320.

5. (2012) Novel Fluoroquinolone (JNJ-Q2).

6. Heeb S, Fletcher MP, Chhabra SR, Diggle SP, Williams P, et al. (2011) Quinolones: From antibiotics to autoinducers. FEMS Microbiol Rev 35: 247-274. 
7. Bolon MK (2011) The newer fluoroquinolones. Med Clin North Am 95: 793-817.

8. Lode $H$ (2010) Safety and tolerability of commonly prescribed oral antibiotics for the treatment of respiratory tract infections. Am J Med 123: S26-S38.

9. Cuzzolin L, Fanos V (2002) Safety of fluoroquinolones in paediatrics. Expert Opin Drug Saf 1: 319-324.

10. Stahlmann R, Lode HM (2013) Risks associated with the therapeutic use of fluoroquinolones. Expert Opin Drug Saf 12: 497-505.

11. (2011) Factive (gemifloxacin mesylate) Tablets, Labeling revision: Supplement approval letter (NDA 21158/S-018).

12. Sirbat D, Saudax E, de Ligny $B H$, Hachet $E$, Raspiller $A$ (1983) Serous macular detachment of the neuro-epithelium and flumequine. J Fr Ophtalmol 6: 829-836.

13. Al-Amri AM (2008) Corneal perforation associated with topically applied gatifloxacin. Cornea $27: 370-371$.

14. Samarakoon N, Harrisberg B, Ell J (2007) Ciprofloxacininduced toxic optic neuropathy. Clin Exp Ophthalmol 35: 102-104.

15. (2015) Retinal detachment. Mayo Clinic.

16. Szarfman A, Chen M, Blum MD (1995) More on fluoroquinolone antibiotics and tendon rupture. $\mathrm{N}$ Engl $\mathrm{J}$ Med 332: 193.

17. (2013) FDA updates warnings for oral and injectable fluoroquinolone antibiotics due to disabling side effects.

18. Etminan M, Forooghian F, Brophy JM, Bird ST, Maberley D (2012) Oral fluoroquinolones and the risk of retinal detachment. JAMA 307: 1414-1419.

19. Rampal S, Kaur R, Sethi R, Singh O, Sood N (2008) Ofloxacin-associated retinopathy in rabbits: Role of oxidative stress. Hum Exp Toxicol 27: 409-415.

20. Choi SY, Lim HA, Yim HW, Park YH (2018) Administration of oral fluoroquinolone and the risk of rhegmatogenous retinal detachment: A nationwide population-based study in Korea. PLoS One 13: e0195563.

21. Kuo SC, Chen YT, Lee YT, Fan NW, Chen SJ, et al. (2014) Association between recent use of fluoroquinolones and rhegmatogenous retinal detachment: A population-based cohort study. Clin Infect Dis 58: 197-203.

22. Pasternak B, Svanstrom H, Melbye M, Hviid A (2013) Association between oral fluoroquinolone use and retinal detachment. JAMA 310: 2184-2190.

23. Raguideau F, Lemaitre M, Dray-Spira R, Zureik M (2016) Association between oral fluoroquinolone use and retinal detachment. JAMA Ophthalmology 134: 415-421.

24. Alves C, Penedones A, Mendes D, Marques FB (2016) A systematic review and meta-analysis of the association between systemic fluoroquinolones and retinal detachment. Acta Ophthalmol 94: e251-e259.

25. Baek YH, Park SJ, Jeong S, Oh IS, Jeong HE, et al. (2018) Signal detection between fluoroquinolone use and the risk of rhegmatogenous retinal detachment: Sequence symmetry analysis using nationwide South Korean healthcare database between 2004 and 2015. Clin Drug Investig 38: 1179-1188.

26. Brett AS (2013) Oral fluoroquinolone use and retinal detachment: Reconciling conflicting findings in observational research. JAMA 310: 2151-2153.
27. Chui CS, Wong IC, Wong LY, Chan EW (2015) Association between oral fluoroquinolone use and the development of retinal detachment: A systematic review and meta-analysis of observational studies. J Antimicrob Chemother 70: $971-$ 978.

28. Daneman N, Lu H, Redelmeier DA (2015) Fluoroquinolones and collagen associated severe adverse events: A longitudinal cohort study. BMJ Open 5: e010077.

29. Douros A, Grabowski K, Stahlmann R (2015) Safety issues and drug-drug interactions with commonly used quinolones. Expert Opin Drug Metab Toxicol 11: 25-39.

30. Eftekhari K, Ghodasra DH, Haynes K, Chen J, Kempen JH, et al. (2014) Risk of retinal tear or detachment with oral fluoroquinolone use: A cohort study. Pharmacoepidemiol Drug Saf 23: 745-752.

31. Fife D, Zhu V, Voss E, Levy-Clarke G, Ryan P (2014) Exposure to oral fluoroquinolones and the risk of retinal detachment: Retrospective analyses of two large healthcare databases. Drug Safety 37: 171-182.

32. Gatti M, Bianchin M, Raschi E, De Ponti F (2020) Assessing the association between fluoroquinolones and emerging adverse drug reactions raised by regulatory agencies: An umbrella review. Eur J Intern Med 75: 60-70.

33. Kapoor KG, Hodge DO, St Sauver JL, Barkmeier AJ (2014) Oral fluoroquinolones and the incidence of rhegmatogenous retinal detachment and symptomatic retinal breaks: A population-based study. Ophthalmology 121: 1269-1273.

34. VanderBeek BL (2016) Oral fluoroquinolones, retinal detachments, and claims database studies. JAMA Ophthalmol 134: 422-423.

35. Huber M, Stahlmann R (2012) The eye as target of adverse ocular drug reactions. Focus on systemic antiinfective therapy. Med Monatsschr Pharm 35: 436-442.

36. Taher M, Alami A, Gravel C, Tsui D, Bjerre L, et al. (2021) Systemic quinolones and risk of retinal detachment I: Analysis of data from the US FDA adverse event reporting system. Expert Opin Drug Saf, 1-8.

37. Taher M, Crispo J, Fortin Y, Moog R, McNair D, et al. (2021) Systemic quinolones and risk of retinal detachment III: A nested case-control study using a US electronic health records database.

38. (2011) Cochrane Handbook for Systematic Reviews of Interventions.

39. SIGN (2014) Search Filters Guidelines. Edinburgh, Scotland.

40. Golder S, Loke YK (2012) Sensitivity and precision of adverse effects search filters in MEDLINE and EMBASE: A case study of fractures with thiazolidinediones. Health Info Libr J 29: 28-38.

41. (2018) Clarivate Analytics. Endnote. Philadelphia, PA, USA.

42. (2014) Review Manager (RevMan). Version 5.3, The Nordic Cochrane Centre, The Cochrane Collaboration, Copenhagen.

43. Moher D, Liberati A, Tetzlaff J, Altman DG, PRISMA Group (2009) Preferred reporting items for systematic reviews and meta-analyses: The PRISMA statement. PLoS Med 6: e1000097.

44. Harmark L, van Grootheest A (2008) Pharmacovigilance: Methods, recent developments and future perspectives. Eur J Clin Pharmacol 64: 743-752. 
45. Coloma P, Trifiro G, Patadia V, Sturkenboom M (2013) Postmarketing safety surveillance: Where does signal detection using electronic healthcare records fit into the big picture? Drug Safety 36: 183-197.

46. Scurti V, Romero M, Tognoni G (2012) A plea for a more epidemiological and patient-oriented pharmacovigilance. Eur J Clin Pharmacol 68: 11-19.

47. Waller P (2010) An introduction to pharmacovigilance. Wiley-Blackwell, Chichester, West Sussex, UK.
48. Friedman L, Furberg C, DeMets D, Reboussin D, Granger C (2015) Fundamentals of clinical trials. ( $5^{\text {th }}$ edn), Springer, New York, USA.

49. Moses C, Celi L, Marshall J (2013) Pharmacovigilance: An active surveillance system to proactively identify risks for adverse events. Popul Health Manag 16: 147-149.

50. (2005) Guidance for Industry: Good Pharmacovigilance Practices and Pharmacoepidemiologic Assessment. 